\title{
Algorithm Model Defining Dimensional Features
}

\section{Friedlander $\mathbf{G M}^{*}$}

Clean Water, Inc, Attn: Gregory Friedlander, 11 S Florida St, Mobile, AL 36606, USA

\begin{abstract}
Energy and Matter is the same thing. Space and Energy are the same thing. Time and change are different. These conclusions give rise to a different view of Atomic Structure and a very specific mathematical model.

The model universe is a Quantum Universe, Quantum solution for quantum point driven, Time Free, Dimension Free, Thermodynamic Free and therefore entropy free.

AuT built this list which is foundational and unique.

1) All dimensions exist together. What this means is:

A) Space has no dimension, photons exist in a single dimension, waves exist only in two; matter 3 and black holes in 4.

B) The definitions of time and space mean that exchanges of information state (ct state) between space, photons occur independent of time and give rise to dimensional features. Exchanges between photons and waves occur independent of time.
\end{abstract}

2) All change is quantum change in response to a single variable leading to the universe existing as a series of quantum states or snapshots. Force and time are effects based on this quantum change.

3) Space is made of the same type of information as all other states (energy, matter, black holes) which are folded from space based on exponential compression/folding.

4) Time and change are different. Changes in the single variable leads to time, but time is not required for that underlying change nor does it affect that change.

5) Time is an effect like force and dimension of the quantum change between states some of which occur without a time-based reference giving rise to features like wave - particle false duality and the impression of charge among other features.

6) Electrons and protons are examples of transitional states (ct3-ct4) between waves (ct3) and neutrons (ct4, molecular states through neutron stars are transitional states between neutrons and black holes (ct5).

Keywords: Algorithm model; Quantum universe

\section{Discussion of the List}

\section{Time and change are different}

a) This has ramifications far down the line. Time is just an effect in the ct3-4-5 interface (electron, proton and black hole matrix). This is why force is so misleading; force is the stacking and unstacking of compression states over time and since ct 1,2 and portions of 3 stacking occur without time, forces like gravity appear to be instantaneous relative to time when they are not relative to quantum change $[1,2]$.

\section{Space and other dimensional features are the same thing}

a) A sub-set is that the mathematical alignment of ct1 states leads to the type of exponential compression set out in the theory as increasing folding of space.

b) All the different dimensional states co-exist. This is a very subtle but important feature of the universe, that non-dimensional, one, two and four dimensional states co-exist with our three dimensional ct4 state; and

c) And the most difficult one to accept, all changes in every point in the universe change together in response to changes in the single variable in a time and dimension free environment.

Algorithm Universe Theory (AuT) defines the structure of not just atomic structure but the underlying spatial matrix and re-defines time as an effect of the building of the matrix like force, dimension itself and the source and effects of Quantum Mechanics and the origin of space time from a non-dimensional matrix.

Algorithm Universe Theory defines the source and effects of Quantum Mechanics and the origin of space time from a nondimensional matrix [3-5].

A mathematical model for generating dimensional features in two equations is presented:

Non-dimensional changes occur as the generation of data points: $\left(\left[-1^{x}\right.\right.$ plus $\left.\left.2 x(-1)^{x-1}\right]\right)$ as $x$ varies from 1 to infinity for points generated. A new starting value of $x$ exists with each new point generated aligned by solution order. Each point has an evolving fuse (or tf-transition fuse) counted down by the same counter. Upon completion a fuse shifts the

*Corresponding author: Friedlander GM, Clean Water, Inc, Attn: Gregory Friedlander, 11 S Florida St, Mobile, AL 36606 USA, Tel: +1- 251-470-0303; E-mail: gmfpc1@gmail.com

Received August 23, 2018; Accepted October 17, 2018; Published October 24 2018

Citation: Friedlander GM (2018) Algorithm Model Defining Dimensional Features J Phys Math 9: 290. doi: 10.4172/2090-0902.1000290

Copyright: (c) 2018 Friedlander GM. This is an open-access article distributed under the terms of the Creative Commons Attribution License, which permits unrestricted use, distribution, and reproduction in any medium, provided the original author and source are credited. 
point to the next higher state, e.g. -3 shifts to 5 after 3 changes in $x$ (a tf=3), 5 changes to -7 after 5 changes in $\mathrm{x}$ with all points changing according to a single variable so that the universe of data points exists in quantum moments of increasingly larger amounts of information with charge varying according to longer fuses;

Dimensional Changes use the data to increase $\mathrm{n}$ as the limit of prior, lower intervening solutions approaches 1 for $2 \mathrm{f}(\mathrm{n})^{2 \mathrm{n}}$ points having alternating positive and negative values. Intervening transitional states, such as the proton and electron for $n=4$ exist without full compression which would result in a neutron.

The interaction of the two derivation equations (both derived sequentially from $-1^{\mathrm{n}}$ ), gives rise to results for observed dimensional features such as force, dimension and time using a single variable. Space becomes the densest substance resulting from the math and all other dimensional forms are derived from folding space mathematically [6-9].

AuT is a very specific, math-based model developed over 5 years based on a critique of Hologram Theory, Relativity, and the Standard Model. Because it takes the approach that the universe is information based, most features in those theories are rejected. A discussion of how they were eliminated appears in Algorithm Universe Theory Compendium (AUTC) Vol 1, second edition and AUTC Vol 2.

AuT relies heavily on mathematics developed 800 years ago and is an extension of the logic work of Parmenides and Zeno dating back 2500 years. To the extent that it covers the work of Marley and Michelson, it comes to the definitive conclusion that they accidentally derailed the proper study of space begun by Descartes 400 years ago, mistakenly rejecting the wrong variable, space, when they should have examined time as the inaccurate variable $[10,11]$.

\section{Differentiating Aut from the Standard Model}

This paper does not go into details of all the mysteries of math which are covered in more detail in Algorithm Universe Theory Compendium Vol1 and 2 which are almost 400 and 800 pages respectively. It does, for example, cover the features which distinguish gravity from other forces; wave particle duality and touches on time dilation.

A comparison of the standard model to the AuT model is beyond the scope of this paper, but there are two books (Algorithm Universe Theory Books 7 and 8 which are part of the second volume of the Compendium) which discuss the findings of the Standard Model in detail. More cursory discussions of string theory, hologram theory and the parallel theory of Processor Universe Theory appear in Volume 1 of the Compendium. AuT is a model and not a theory.

A cursory examination of the differences from other models is given here.

I. A two-equation solution

1) One generating changeable data points

2) One generating and destroying dimensional features for the solutions and order of solutions of the data points

II. Differentiation of change from time and treating time as an effect like force and dimension.

III. Separating time based dimensional features and non-timebased features

IV. Showing all dimensional states exist together
V. Showing the universe is a single entangled strand of solutions separated by solution order at the first ct level.

VI. Showing net compression/decompression states as the observed effect locally and universally. math.

VII. Showing dimension, force and time are effects of an underlying

VIII. The counter between inflection points is based on the fpix equation which grows longer which each value of $x$, but apparently restarts for each new point created with each change in $\mathrm{x}$. This fpix state change (from positive to negative) after a fuse count of increasing fpix count defines all forces and anti-forces and all ct states as being compressive or non-compressive and entropy or the alternative, gravity or the alternative dark energy.

VIV. The universe existing as quantum moments/snapshots in response to a single variable.

$\mathrm{X}$. Force and time being the effect of loading and unloading "virtual' information arms.

XI. $\operatorname{Pi}(\mathrm{x})$ is defined as fpix for the range from 2 to a maximum of 5 of for $\mathrm{N}$ of $\mathrm{N} / \mathrm{F}$ (pix)] summed over the number of points for the ct state under consideration. For example, there are $10^{16 *} 6^{8 *} 4^{4} \mathrm{ct} 2$ states giving rise to dimension for ct4. "Warping of space" is just the inclusion of an additional dimensional feature defined by $\mathrm{N}=5$ for pi adding up to $16^{32}$ ct2 states for each ct4 state.

XII. showing that space is the fundamental structural component of the universe and that black holes are merely the highest visible dimensional compression state.

\section{Notes on the Results of Differentiation}

I. The universe can be thought of as a string of quantum positive or negative values which change polarity according to growing fpix solutions with those changes resulting in constant changes from compression/folding to decompression/unfolding and back again based on this pairing of opposite sates and unmatched states trapped within the folds created.

II. If time is treated only as an effect, it can largely be replaced in all manipulative equations to get more efficient results. Quantum change (comparable but different from time) is put forward which yields values for force, dimension and clock time based on dimensional changes occurring in a time free environment based on the idea that at any quantum moment (value of $\mathrm{x}$ ) the universe would be fixed, no clock time, force or velocity. The model solves for time, force, dimension and velocity in the absence of time to provide perspective.

III. Dimensional states co-exist. Space is non-dimensional, photons have one, waves two and matter 3 , for example. We only perceive these as separate dimensions because of our perspective. An analogy would be looking at a "wave bus" with blackened windows moving down a street. Whatever happens to the photons within the bus can happen at any speed, we're stuck with just seeing the outside dimension of the bus, the inside dimension of the bus is invisible to us.

The traditional view is the view of a circle passing through a line looking like a point and then two dots increasingly spaced on the line before they begin to come together to another point.

We see zero, one and two-dimensional features as if they were 3 dimensional; so too we see change rates as if they are limited by our rate change, when they are not. 


\section{IV. "Space and matter are the same thing."}

A corollary is that the most solid thing in the universe is ct1, space, in terms of having nothing separating it. You cannot pass through space, you can only move with it and for that reason a different approach to space based on its density will yield better velocity and time-based results.

Space must move everything else around.

We can convert matter to space and we "see" the conversion via the intermediary states of waves and photons as velocity [12].

\section{Dimensional Features of the Universe}

Before we get to the equations, it is helpful to see what the result of the equations yields in terms of dimensional features.

\section{Figure line compression}

Compression builds from non-dimensional space (ct1) to black holes (ct5) by increased folding. In so doing, the line becomes partially folded and then folds at an exponentially increasing rate.

The movement of ctl in straightening out through highly compressed ct3-ct4-ct5 states involves dragging ct1 states into and through and out of these higher compression states substituting in the process or removing or adding free states in the process (Figure 1).

\section{Figure electron-proton compression}

If we look at a single neutron (above), we can see it is built from ct3 states (far left) which align along the information arms of the ct 4 state; building from wave energy (ct3) to transitional electron/proton states and then to matter (ct4) as the number of trapped states falls below a certain level (Figure 2).

If each arm builds there is a ratio of 1:100 at 7:9 (7 information arms for the electron to 9 arms for the proton) which is as close as you get to a ratio of split arms but in this case, you only need 18 times the amount of information in the electron bundle (the hinge or entrapped states of ct3, 2 and 1) vs. the electron itself. If you take into account the inadequacy of mass as a way of comparing the two, you can arguably change the 18 to 16 and now the two (electron to proton) can be viewed as the electron being the uneven c3-ct4 state at 7 arms, the proton being the uneven ct3-ct4 at 9 arms and the electron bundle (electron cloud) being 16 times the electron itself so that there are $2^{\mathrm{n}}$ times the stable information lower states $(n=4)$ (Figure 3 ).

As energy is added Mass increases not because of actual movement, but because the proximate concentration of ct 1 to $\mathrm{ct} 3$ transitional states necessary for ct 3 alignment along ct 4 carrier arms.

\section{Helium ct4-ct5 Transition States}

Helium formation is very different from the formation of a neutron. The alignment of the two Proton-electron pairs represents ct5 information arm loading, albeit at its earliest features. If carried forward it would eventual go from transient ct4-ct5 features to a black hole (Figure 4).

What this simplified figure represents is two protons and two electrons; or Helium. It follows a single quantum of space identified initially as ctla. This unentangled ctla is being pulled towards linearity

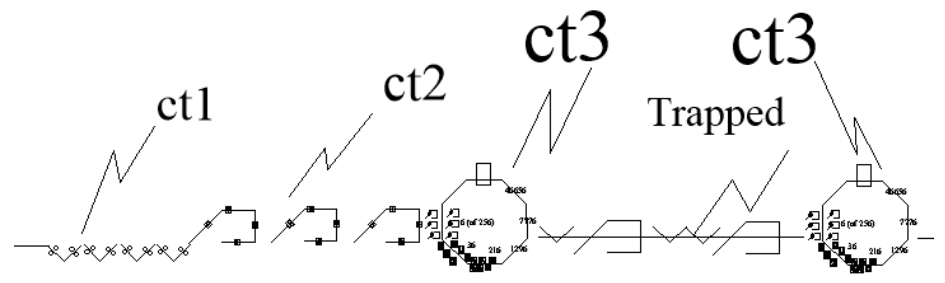

Figure 1: Compression builds from non-dimensional space (ct1) to black holes (ct5) by increased folding. In so doing, the line becomes partially folded and then folds at an exponentially increasing rate.

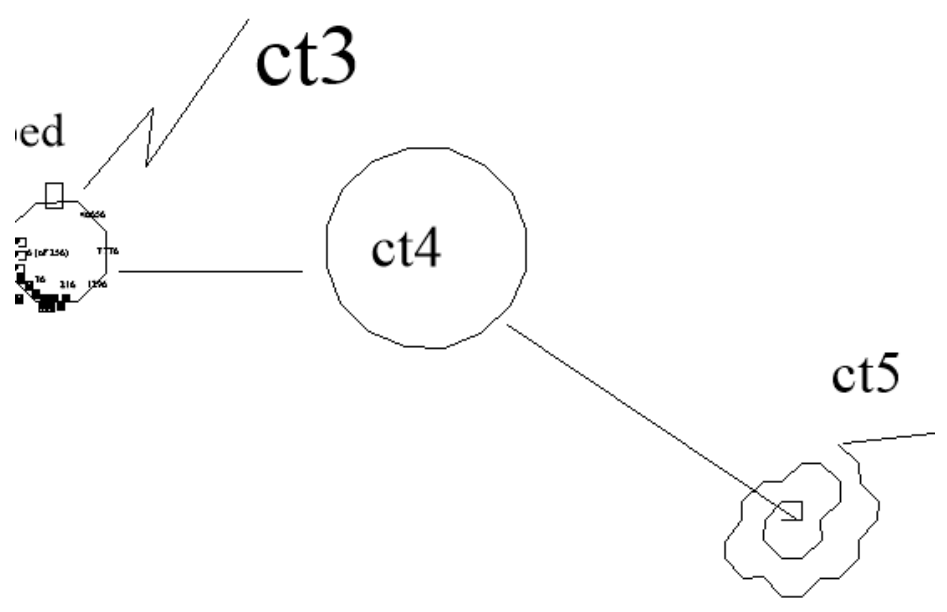

Figure 2: The movement of ct1 in straightening out through highly compressed ct3-ct4-ct5 states involves dragging ct1 states into and through and out of these higher compression states substituting in the process or removing or adding free states in the process. 


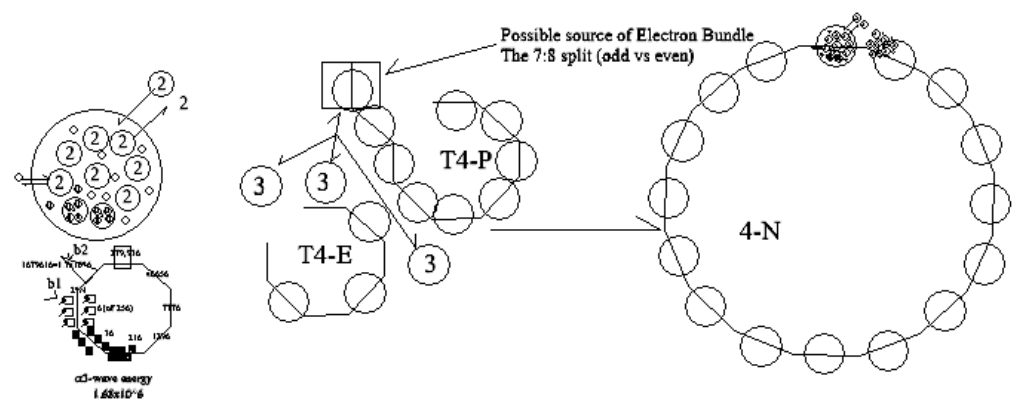

Figure 3: Figure showing electron-proton compression.

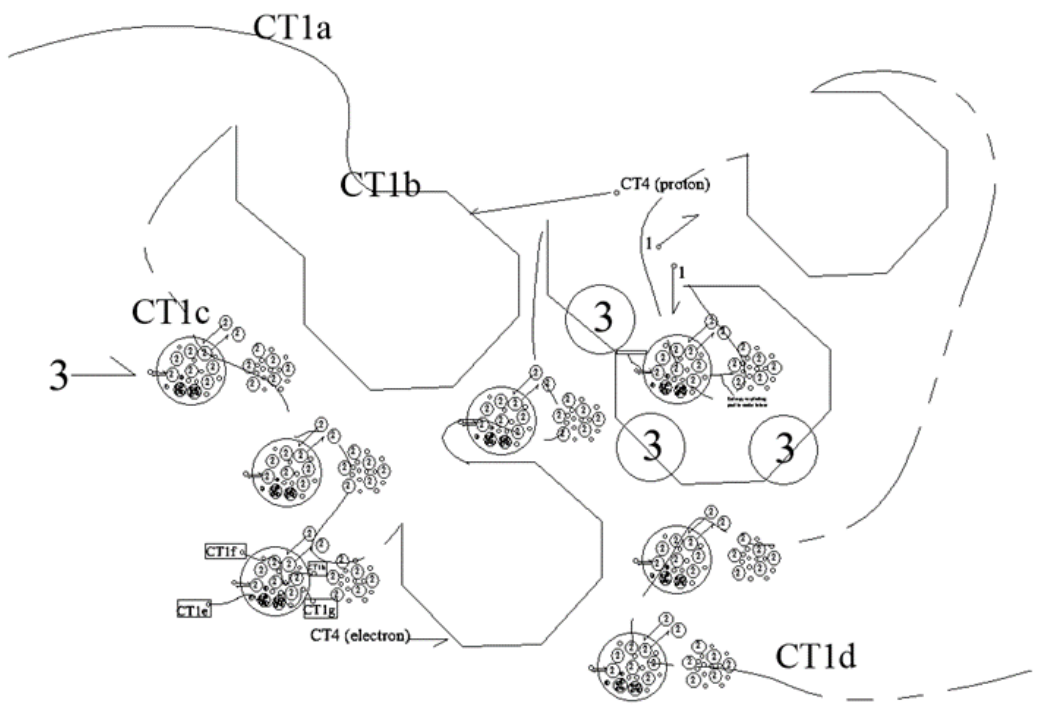

Figure 4: Helium the line moving through represents that path of a single ct1 of the possible $10^{\wedge} 38$ separate ct1 states possible within each neutron and building toward a multiple of $16^{\wedge} 32$ for molecular structures.

as the net value of changes towards or away from compression (entangled) changes. By definition, this ctla defines a tiny part of the separation of higher ct states (ct2, 3, 4, etc.).

As ctla is pulled by decompression it gets dragged through the knots of higher compression and at ctlb it is shown entering the matrix of a proton. At this point, CT1b, it may well substitute for another ct1 state and become part of the compression, but it no longer separates the Helium matrix from surrounding space, it has become part of the matrix and therefore becomes a feature of time and not separation. At ctlc our entangled ctl is shown being dragged through and thereby altering a free ct 3 state which is made up of ct 2 states which in turn are made up of ct1 states [13,14].

Given the scale of compression, this ctl state has only a minor effect on the organization of the ct3. Each ct 3 is made up of $4.3 \times 10^{8} \mathrm{ct} 1$ states and perhaps as many free ct1 states like this one.

While this drawing follows a single ct 1 , the number of ct 1 changes occurring over even a small number of changes in $\mathrm{x}$ is very large and there are $10^{44}$ changes in $\mathrm{x}$ every second so this is a very dramatic and dynamic event at the scale where we observe time; but at the quantum level this is non-dimensional and only reflects ctl states gradually changing from positive to negative values and being solved in an order. The speed of the changes in position of ctl are a function of the rate at which the regional changes from positive to negative occur and how these changes interact with the changes in charge of this ctl.

While an isolated matrix is shown, the overall matrix of a planet with helium as a part, would share a general commonality of time because the ct5 information arms holding molecular structures together allow for a dispersion of the electron bundle of trapped ct3 states (Figure 5).

\section{Fundamental Equations Giving Rise to Graphic Results}

The universe can be described by several related equations:

$\Delta \neq \mathrm{t}$.

This equation points out that change is defined by quantum change and that time is not equal to that change. This has ramifications far down the line. Time is just an effect in the ct3-4-5 interface (electron, proton and black hole matrix). Therefore force is misleading; force is the stacking and unstacking of compression states over time and since ct 1,2 and portions of 3 stacking occur without time, forces like gravity appear to be instantaneous relative to time when they are not relative to quantum change.

Mass can be defined as space: $c t 1=f u n c(x) m$.

This equation sets out that space and mass are the same. The equation is complicated by the massive number of adjacent solutions 


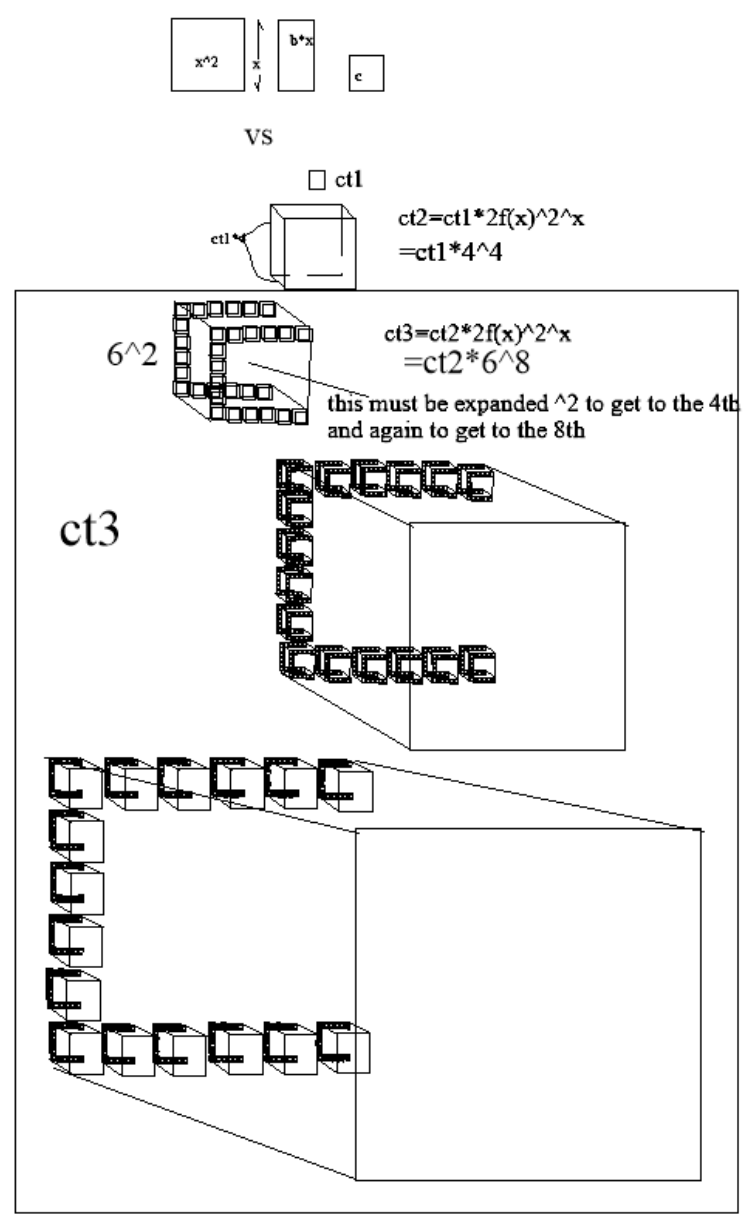

Figure 5: A comparison between the standard polynomial compression is shown below for box solutions.

that must be solved to describe a single neutron.

Breaking this down we get:

$$
\mathrm{f}\left(-1^{\mathrm{x}}\right)=\left(\left[-1^{\mathrm{x}} \text { plus } 2 \mathrm{x}(-1)^{\mathrm{x}-1}\right]\right)=\text { fpix }
$$

which is the equation that defines how we create differentiation in a universe where -1 is the only basic feature. The only solution which works for this equation is where $\mathrm{x}=0$.

The next equation derived in the same fashion is:

$$
\sum \text { fpix }=\text { Matrix }\left[2 f(x)^{2^{x}}\right]
$$

This equation represents that over any value of $x$, the sum of all of the fpix solutions yield a matrix of compression solutions that define the universe for that value of $\mathrm{x}$ [16].

If Space and other dimensional features are the same thing, the relationship has to be defined and it can be by the Matrix equation:

$$
\text { Matrix }\left[2 f(x)^{2^{x}}\right]
$$

This equation is positional requiring the alignment of solutions of opposite polarity:

The best guess for how this compression or ratio of state changes occurs is based on the comparison of -1 and 1 dimensional states which exist by virtue of $-1^{\mathrm{x}}$ being the fundamental data generation equation (Table 1).

\begin{tabular}{|c|c|c|c|c|}
\hline & $\mathbf{n}$ & Siny & siny & siny \\
\hline $32 / 27 / 8$ & & $2^{*} \mathrm{y} /\left(\mathrm{pi}-1^{(2 \mathrm{n}+1)}\right)$ & $2^{*} \mathrm{y} /\left(\mathrm{pi} 1^{(2 \mathrm{n}+1)}\right)$ & $2^{*} \mathrm{y} /\left(\mathrm{pi} 2^{(2 \mathrm{n}+1)}\right)$ \\
\hline 0.148148 & $\mathrm{y}=1$ & $\mathrm{pi} \mathrm{of}-1 / \mathrm{ac} 2=-1$ & 0.148148 & $1.19 \mathrm{E}+00$ \\
\hline 1 & for $\mathrm{n}=0-(-1)->$ & -0.44444 & 0.444444 & 1.7777778 \\
\hline 1 & for $\mathrm{n}=1$ & -1 & 1 & 1 \\
\hline
\end{tabular}

Table 1: ratio of $\sin (\mathrm{pi}-1)$ to $\sin (\mathrm{pi} 1)$.

This chart shows that the ratio of the sin for $\mathrm{p}-1$ and pi1 is $256: 27$. It also shows the ratio from $\sin ($ pi1) to $\sin (\mathrm{pi} 2)$ to one place to be 8 times that ratio. These equations ignore the infinite series solutions required in 3 dimensions because there is only one dimension represented between the two zero dimensional states ( -1 and 1$)$ and the one dimensional photonic state (pi for 2).

Utilizing this ratio, the information arms represented by $2^{\mathrm{x}}$ in the Matrix equation can be represented by $2^{\mathrm{x}}: 3^{(\mathrm{x}-1)}$ which provides a satisfactory explanation of the entrapped information within each compressed state which might also be called hinge information in the ct1 to ct2 compression state (Table 2).

The first column represents the ct state as well as the numerator of pi for that state. The second column is two times the Fibonacci number for the ct state.

The third column is the number of "folds" or "information arms" for the ct state in question. 


\begin{tabular}{|c|c|c|c|c|}
\hline Information & $\mathbf{2}^{\mathbf{*}} \mathbf{( n )}$ & $\mathbf{2}^{\mathbf{n}}$ & $\mathbf{2 f}^{\mathbf{n}(\mathbf{n})^{2 \mathbf{n}}}$ & Dim State \\
\hline \multicolumn{3}{|c|}{ N-CT State } & & Next lower states \\
\hline 1 & 2 & 2 & 4 & Space-0 \\
\hline 2 & 4 & 4 & 256 & Photon-1 \\
\hline 3 & 6 & 8 & 1679616 & Wave-2 \\
\hline 4 & 10 & 16 & $1.00 \mathrm{E}+16$ & Matter-3 \\
\hline 5 & 16 & 32 & $3.40 \mathrm{E}+38$ & Black hole-4 \\
\hline
\end{tabular}

Table 2: Chart of compression states.

The fourth column is the number of matched states for each ct or compression state where they are matched by positive value of fpix to negative value of fpix [17].

So, for example, for ct 2 , there are 128 positive and 128 negative states which are matched of ct1 (space) for a total of 256 aligned on 4 virtual information arms. The information arms may be equally loaded 64 on each arm or may be loaded as a factor 4:16:64:256. The 5 th column is the dimension generated from 0 to 4 .

A comparison is given between the standard polynomial compression is shown below for box solutions.

While the first transition from ct1 to ct2 is fairly easy to represent, the second transition from ct 2 to ct 3 is more challenging without an extremely large canvas and for perspective the scale is changed for every transition after the ct 1 to $\mathrm{ct} 2$ transition.

The big box shows the ct 3 transition.

\section{Limit Equations}

The collapse from one state to the prior state is the limit equation:

Which represents the collapse of one state into the next higher state (or the dissolution of the higher state into the next lower state) as the number of intervening unmatched states (I) approaches the allowed number of Hinge or unmatched states.

Using $3^{\text {n-1 }}$ the hinge states may drop to either 9 per hinge or may increase as a factor 3:9:27.

The values of the quantum points making up these ct states vary independent of one another, but based on a single variable: $\left(\left[-1^{x}\right.\right.$ plus $\left.\left.2 x(-1)^{x-1}\right]\right)$ as $x$ varies from 1 to infinity for points generated with a new starting value of $\mathrm{x}$ with one new point generated for each change in $\mathrm{x}$ aligned by solution order; each point has a fuse counted down by the same counter upon completion of such fuse shifts the point to the next higher state, e.g. -3 shifts to 5 after 3 changes in $x, 5$ changes to -7 after 5 changes in $\mathrm{x}$ with all points changing according to a single variable.

The resulting pattern of solutions looks something like this where even the first 9 changes in $\mathrm{x}$ leads to a very complicated result (Table 3.1).

Which for the first 20 results leads to a result something like this where the shift from a net expanding to contracting solution occurs relatively quickly but over an increasingly long period of changes in $\mathrm{x}$. It is worth nothing that you need a minimum of $256+27$ solutions to generate a single photon with the proper alignment for this model to begin to yield dimensional results of the type we experience (Table 3.2).

Each equation evolves from a fairly simple expedient of memorizing the prior solution and the results of the prior solution which get you sequentially from $-1^{x}$ to fpix to $\left(2 f(x)^{2 x}\right)+\left(3^{n-1}\right)$ by remembering solutions and solution order (Figure 6).

The mathematical alignment of ct 1 states leads to the type of exponential compression set out in the theory as increasing folding of space.

\begin{tabular}{|c|c|c|c|c|c|c|c|}
\hline $\mathbf{x}$ & 0 & & 0 & 1 & 2 & 3 & 4 \\
\hline & 1 & & -1 & & & & \\
\hline & 2 & -1 & 1 & -3 & & & \\
\hline & 3 & -1 & 2 & -3 & -3 & & \\
\hline & 4 & -1 & 2 & -3 & -3 & -3 & \\
\hline & 5 & -1 & 2 & -3 & -3 & -7 & 9 \\
\hline & 6 & -1 & 2 & -3 & -3 & -7 & 9 \\
\hline & 7 & -1 & 2 & -3 & -3 & -7 & 9 \\
\hline & 8 & -1 & 2 & -3 & -3 & -7 & 9 \\
\hline & 9 & -1 & 2 & -3 & -3 & -7 & 9 \\
\hline & 10 & -1 & 2 & -3 & -3 & -7 & 9 \\
\hline & 11 & -1 & 2 & -3 & -3 & -7 & 9 \\
\hline & 12 & -1 & 2 & -3 & -3 & -7 & 9 \\
\hline & 13 & -1 & 2 & -3 & -3 & -7 & 9 \\
\hline & 14 & -1 & 2 & -3 & -3 & -7 & 9 \\
\hline & 15 & -1 & 2 & -3 & -3 & -7 & 9 \\
\hline & 16 & -1 & 2 & -3 & -3 & -7 & 9 \\
\hline & 17 & -1 & 2 & -3 & -3 & -7 & 9 \\
\hline & 18 & -1 & 2 & -3 & -3 & -7 & 9 \\
\hline & 19 & -1 & 2 & -3 & -3 & -7 & 9 \\
\hline & 20 & -1 & 2 & -3 & -3 & -7 & 9 \\
\hline & 21 & -1 & 2 & -3 & -3 & -7 & 9 \\
\hline & 22 & -1 & 2 & -3 & -3 & -7 & 9 \\
\hline & 23 & -1 & 2 & -3 & -3 & -7 & 9 \\
\hline & 24 & -1 & 2 & -3 & -3 & -7 & 9 \\
\hline & 25 & -1 & 2 & -3 & -3 & -7 & 9 \\
\hline & 26 & -1 & 2 & -3 & -3 & -7 & 9 \\
\hline & 27 & -1 & 2 & -3 & -3 & -7 & 9 \\
\hline
\end{tabular}

Table 3.1: Table representative memorized solutions-part 1.

\begin{tabular}{|c|c|c|c|c|c|c|c|}
\hline $\mathbf{5}$ & $\mathbf{6}$ & $\mathbf{7}$ & $\mathbf{8}$ & $\mathbf{9}$ & $\mathbf{1 0}$ & $\mathbf{1 1}$ & $\mathbf{1 2}$ \\
\hline-11 & & & & & & & \\
\hline-11 & 13 & & & & & & \\
\hline-11 & 13 & -15 & & & & & \\
\hline-11 & 13 & -15 & 17 & & & & \\
\hline-11 & 13 & -15 & 17 & -19 & & & \\
\hline-11 & 13 & -15 & 17 & -19 & 21 & & \\
\hline-11 & 13 & -15 & 17 & -19 & 21 & -23 & \\
\hline-11 & 13 & -15 & 17 & -19 & 21 & -23 & 25 \\
\hline-11 & 13 & -15 & 17 & -19 & 21 & -23 & 25 \\
\hline-11 & 13 & -15 & 17 & -19 & 21 & -23 & 25 \\
\hline-11 & 13 & -15 & 17 & -19 & 21 & -23 & 25 \\
\hline-11 & 13 & -15 & 17 & -19 & 21 & -23 & 25 \\
\hline-11 & 13 & -15 & 17 & -19 & 21 & -23 & 25 \\
\hline-11 & 13 & -15 & 17 & -19 & 21 & -23 & 25 \\
\hline-11 & 13 & -15 & 17 & -19 & 21 & -23 & 25 \\
\hline-11 & 13 & -15 & 17 & -19 & 21 & -23 & 25 \\
\hline-11 & 13 & -15 & 17 & -19 & 21 & -23 & 25 \\
\hline-11 & 13 & -15 & 17 & -19 & 21 & -23 & 25 \\
\hline-11 & 13 & -15 & 17 & -19 & 21 & -23 & 25 \\
\hline-11 & 13 & -15 & 17 & -19 & 21 & -23 & 25 \\
\hline-11 & 13 & -15 & 17 & -19 & 21 & -23 & 25 \\
\hline-11 & 13 & -15 & 17 & -19 & 21 & -23 & 25 \\
\hline & 13 & & & & \\
\hline
\end{tabular}

Table 3.2: Table representative memorized solutions-part 2 .

Quantum change yields values for force, dimension and time based on dimensional changes based on the idea that at any quantum moment the universe is fixed and can be solved for time, force, dimension and potential velocity in the absence of time (Table 3.3).

Dimensional states co-exist. Space is non-dimensional, photons have one, waves two and matter 3, black holes 4 . We separate 


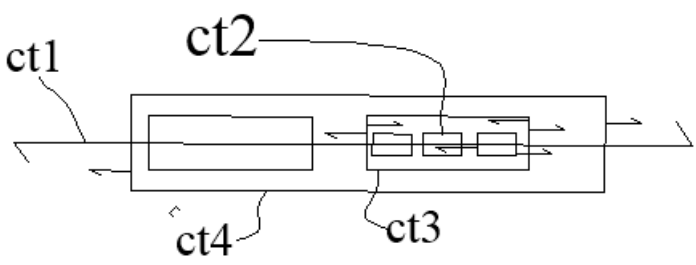

Figure 6: Concept drawing of different compression/decompression levels.

\begin{tabular}{|l|l|l|l|l|l|l|}
\hline $\mathbf{1 3}$ & $\mathbf{1 4}$ & $\mathbf{1 5}$ & $\mathbf{1 6}$ & $\mathbf{1 7}$ & $\mathbf{1 8}$ & $\mathbf{1 9}$ \\
\hline-27 & & & & & & \\
\hline-27 & 29 & & & & & \\
\hline-27 & 29 & -31 & & & & \\
\hline-27 & 29 & -31 & 33 & & & \\
\hline-27 & 29 & -31 & 33 & -35 & & \\
\hline-27 & 29 & -31 & 33 & -35 & 37 & \\
\hline-27 & 29 & -31 & 33 & -35 & 37 & -39 \\
\hline-27 & 29 & -31 & 33 & -35 & 37 & -39 \\
\hline-27 & 29 & -31 & 33 & -35 & 37 & -39 \\
\hline-27 & 29 & -31 & 33 & -35 & 37 & -39 \\
\hline-27 & 29 & -31 & 33 & -35 & 37 & -39 \\
\hline-27 & 29 & -31 & 33 & -35 & 37 & -39 \\
\hline-27 & 29 & -31 & 33 & -35 & 37 & -39 \\
\hline-27 & 29 & -31 & 33 & -35 & 37 & -39 \\
\hline
\end{tabular}

Table 3.3: Table representative memorized solutions-part 3.

dimensions through perspective. Whatever happens to photons within waves is invisible, so we see the net result as duality. We see zero, one and two-dimensional features as if they were 3 dimensional. We see change limited by wave state rate change which distorts our perception of the other, lower states changes.

All states maintain their dimensional status. We see the constant interaction of space and photons as movement. Movement is nondimensional ct 1 space decompressing and unwinding as trapped ct 1 states escaping from a folded matrix of higher ct states. Ct2 photons decompress from ct3 wave energy and wave energy from ct 4 matter. Protons and electrons are ct 4 transitional states. Ct5 is what we call black holes and the transitional states of ct5 are what we call molecular structures (Table 4).

A. The universe is defined by quantum changes in a single variable giving rise to quantum moments or quantum instants for each quantum state for the entire universe.

B. Time is a secondary phenomenon reflecting the difference between velocity; ct1 entrapment and release from the ct3-ct4 transitional state folds (proton-electron is a transitional ct4 neutron state).

C. Space, photons, wave energy, neutrons and black hole material define the visible states of compression. Electrons, proton, neutrons and photons share ct3 wave energy as a transitional state giving rise to perceived wave properties of those solution. Electrons and Protons are transitional states to Neutrons. Dimension arises as a result of the $\mathrm{f}$-series base equation $\left(2^{\star} f(n)\right)^{2 n}$ where $f(n)$ is the Fibonacci number for $n$ and where each dimension adds a place in the number of $F(n)$ solutions beginning with 2 places for the first dimension

D. Force is the loading and unloading of the $2^{\mathrm{n}}$ compression states which are also called information arms as a visual reference in a time free environment as observed over time. While it is impossible to scale the constant movement of states towards or away from compressed states, it can be graphically represented understanding that the ct1 and 2 state transitions are unobservable from our ct3-4-5 viewpoint of changing ct3 states and this model suggests that photons, ct2, are far different from what we envisioned, the background radiation otherwise ascribed to the big bang being one possible effect.

1. The transitional state (partial loading and unloading of information arms) from ct1(space) to ct2(photons) is reflected by gravity and the ct 2 to ct 3 transition gives rise to electro-magnetism. The strong and weak force reflect ct3-ct4 arm loading and ct4-ct5 arm loading although transitional ct3-ct4 states also load as protons onto ct5 information arms (Table 5.1).

2. AuT suggests a result for quantum state changes in the universe can be determined free of thermodynamics, including entropy, and uncertainty. It explains how dimension and space time arise from a non-dimensional framework and how different dimensional states exist independent of one another but are joined by way of being separate compression states (Table 5.2).

\begin{tabular}{|c|c|}
\hline Big bangs (very small ones) & Diff between results \\
\hline-1 & \\
\hline-3 & 2 \\
\hline-5 & 2 \\
\hline-8 & 3 \\
\hline-3 & -5 \\
\hline-14 & 11 \\
\hline-1 & -13 \\
\hline-16 & 15 \\
\hline 1 & -17 \\
\hline-18 & 19 \\
\hline 3 & -21 \\
\hline-20 & 23 \\
\hline 5 & -25 \\
\hline-22 & 27 \\
\hline 7 & -29 \\
\hline-24 & 31 \\
\hline 9 & -33 \\
\hline-26 & 35 \\
\hline 11 & -37 \\
\hline-28 & 39 \\
\hline 13 & -41 \\
\hline-30 & 43 \\
\hline 15 & -45 \\
\hline-32 & 47 \\
\hline 17 & -49 \\
\hline
\end{tabular}

Table 4: Changing net inflection points from prior table.

\begin{tabular}{|l|l|l|l|l|l|l|l|l|l|}
\hline $\mathbf{X}$ & ABS Sum & & & & & & & \\
\hline & Also Total fpix & & & & & & & \\
\hline & 0 & & & & & & & & \\
\hline 1 & 3 & -3 & & & & & & & \\
\hline 2 & 8 & 5 & -3 & & & & & & \\
\hline 3 & 15 & -7 & 5 & -3 & & & & & \\
\hline 4 & 24 & 9 & -7 & 5 & -3 & & & & \\
\hline 5 & 35 & -11 & 9 & -7 & 5 & -3 & & & \\
\hline 6 & 48 & 13 & -11 & 9 & -7 & 5 & -3 & & \\
\hline 7 & 63 & -15 & 13 & -11 & 9 & -7 & 5 & -3 & \\
\hline 8 & 80 & 17 & -15 & 13 & -11 & 9 & -7 & 5 & -3 \\
\hline
\end{tabular}

Table 5.1: Fibonacci Changes from summed solutions: part 1. 


\section{Compression}

The representation of information arms and compression below reflects the model for the compression model reflecting the equation $2 \mathrm{f}(\mathrm{n})^{2 \mathrm{n}}$ (Figure 7).

What you can see (fairly clearly at the ct2 level) is that there are packets of information. At ct1, there are only 4 total "bits", but at each arm of ct2, the number of these bits increases by a factor of 2 , so arm one has 4; $\operatorname{arm} 2,16 ; \operatorname{arm~3,32}$ and $\operatorname{arm} 4,256$ with these very large packets being compressed at ct 3 and ct 4 . Ct 3 shows this and it's already so high $\left(1.68 \times 10^{6}\right)$ of total compression and that significant history formed by transitions are possible.

Net rates for decompression of the universe and locally for our galaxy and planet are discussed in the compendium.

\section{The True Nature of Dimension}

\section{Science vs. pseudo-science}

Criticism is perhaps the strongest clarifier of knowledge. I am here seeking Criticism but also indulgence and a willingness to either ignore

\begin{tabular}{|c|c|c|}
\hline $\begin{array}{c}4^{*} \times \text { separation from } \\
\text { prior }\end{array}$ & $\begin{array}{c}\text { Regular sum each } \\
\text { column d-k }\end{array}$ & Difference from prior $\mathbf{m}$ \\
\hline 4 & -3 & 5 \\
\hline 8 & 2 & -7 \\
\hline 12 & -5 & 9 \\
\hline 16 & 4 & -11 \\
\hline 20 & -7 & 13 \\
\hline 24 & 6 & -15 \\
\hline 28 & -9 & 17 \\
\hline 32 & 8 & \\
\hline
\end{tabular}

Table 5.2: Fibonacci Changes from summed solutions: part-2. me or to have a discussion which borders on radical, but I would submit it is not radical. As long as a theory is "firmly" founded in mathematics, I argue it is unlikely to be pseudo science. AuT is math. The suggested application of math is not inconsistent with leading theories of physics like Hologram and Simulation theory. Specifically, Peter Hanggi has discussed the universe as "a giant quantum information processor instead of a physical thing." AuT provides a specific model for the operation of the information processor in two equations.

AuT lays down in the face of observations and says, we have to rethink so we can accept and not force the math. One of the early suggestions which was made was that that logic of AuT (actually a predecessor NLT) "sounded like Zeno's work" which was 2500 years old. That is true. The logical problem which Parmenides and Zeno dealt with had to do with the infinite divisibility of space and the resulting paradox, the so called "Zeno's paradox."

AuT provides a solution to the paradox (AUTC).

\section{Terms}

1. X-The count of the universe, counting in sequential quantum whole numbers $(1,2,3$, etc). Each number represents a quantum instant for the entire universe.

2. Quantum points, quantum data points: Individual bits of fpix information which make up the universe. It is estimated there are at least $10^{100}$ of these defining everything from space to black holes. Each has a positive or negative charge for any value of $\mathrm{x}$.

3. Transition fuses (tf): The number of changes in $\mathrm{x}$ between charge changes for quantum points. The direction of changes at the ct2-ct3 level is seen as traditional charge.

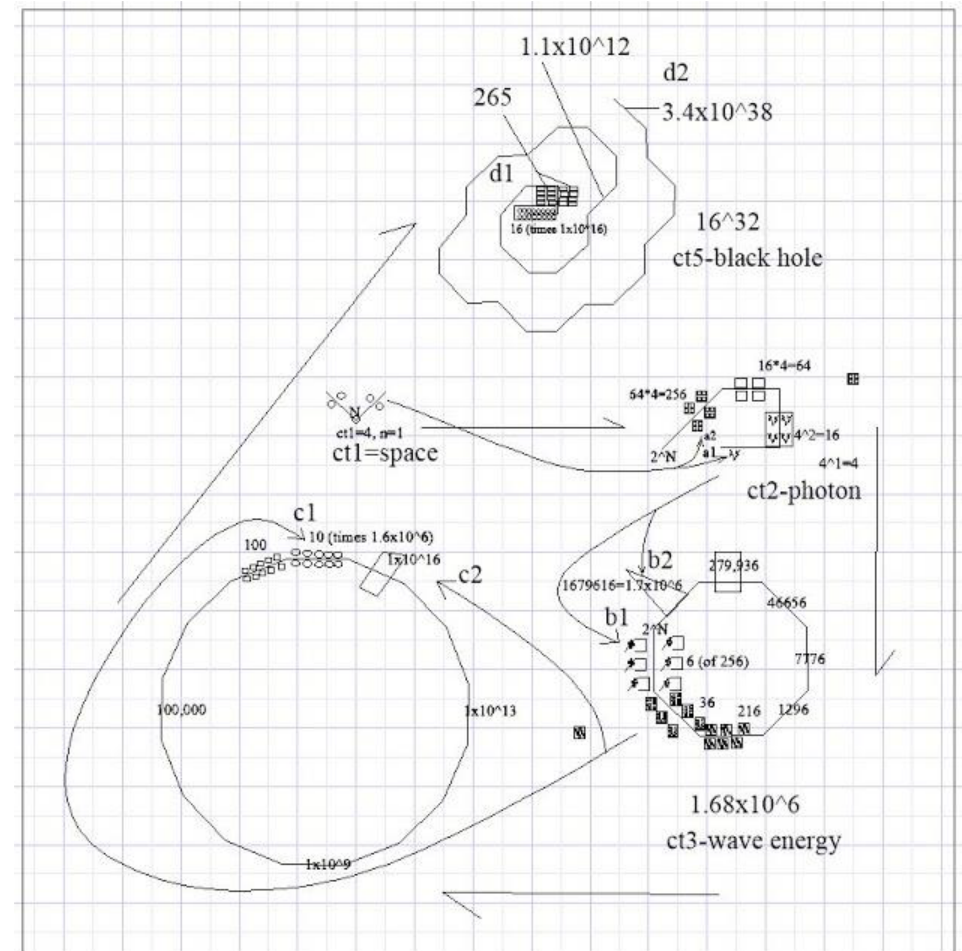

Figure 7: The representation of information arms and compression below reflects the model for the compression model reflecting the equation $2 f(n){ }^{\wedge} 2^{\wedge} n$. 
4. Fpix: the denominator of pi and an equation that separates quantum points of the universe by transition fuses.

5. F-series: The Fibonacci series $\mathrm{f}(\mathrm{n})$; f-series compression refers to two times this number.

6. Exponential compression $2^{\text {n }}$ (see information arms).

7. Place: Also compression sate defined by the f-series raised to the exponential compression number which defines dimension.

8. Information arms-the number of folds represented by exponential compression based on pairing positive and negative lower ct states.

9. Transitional states: Compression states where the information arms are partially filled or separated by trapped states. Two examples are protons and electrons. Transitional states can be described as the transition between a lower state and a higher state. A ct3-ct4 transitional state, such as the electron and proton, are transitional states between waves and neutrons with too many intervening transitional states to fully collapse into neutrons.

10. Force: Changes over values of $x$ in the filling of information arms viewed from a time based analysis. Electro-magnetism is theorized to be the loading and unloading of ct2 states onto ct3 information arms; gravity ct1 loading/unloading onto ct2 information arms. Since the net effect of time free changes is observed from a time based perspective, we "see" these time independent changes as forces.

11. Light speed: The rate change represented by a single ct2 state relative to a ct 3 state viewed from a time based perspective.

12. Time: The ratio of ctl states passing within a ct3-ct4-ct5 transitional state to $\mathrm{ct} 1$ states changing outside of the ct3-ct4-ct5 transitional state. This ratio is the source of velocity time dilation. The movement of ctl states within the ct3-ct4 transitional state alter the arrangement of the ct3 wave states captured between the proton and the electron altering the history of points within the transitional state and the comparison of one collection of points to subsequent arrangements of the same points creates history.

13. Electron bundle: The waves states within ct 3 -ct 4 and ct4-ct5 transitional states which hold the history for a regional mass.

14. Gravity: The force created by non-dimensional ct1 solutions being "loaded" onto information arms of ct2.
15. Dark energy (anti-gravity)-the force created by nondimensional ct1 solutions being unloaded from information arms of ct 2 . This may include releasing ct 1 states trapped within a higher ct state matrix.

16. Ct1-space defined by fpix sequential solutions

17. Ct2-photons: The first compression state, unique as coming from a non-dimensional state to a one dimensional state. Note that the transition between ct1, ct 2 and ct 3 occur below the level of time and the transitions are therefore partially hidden from a time-based analysis.

18. Ct3: Waves.

19. Ct4-neutrons: Note that neutrons do not allow for significant passing of ct 1 states within the ct4 state due to the compressed nature of the neutrons, so time does not exist within neutrons. This is the source of gravitational time dilation.

20. Ct5-black holes: Molecular states are ct4-ct5 transitional states.

21. Ct6 and beyond: theorized higher compression states that will form when the total amount of information in the universe is sufficient.

22. The big bang: A misnomer referring to the time when the collection of compression solutions of ctl states into higher ct states went from a net positive compression state to a net negative decompressing state reflecting a greater release of ctl states from higher states than the corresponding entrapment. We are approximately 13 billion years ( 13 billion $\times 10^{44}$ changes in $\mathrm{x}$ ) from the latest inflection point and approximately 7 billion years from the next inflection point where the universe will begin to contract again.

\section{CT4 State Interaction and Time}

Look at a visual, every compression state is surrounded by other compression states: Below is an alternative view of Hydrogen or it can also be seen as two protons of Helium.

You have here 2 neutrons (ct4) surrounded by layers of ct3 (waves), ct2 (photons) and ct1 space. This is also conceptually how an electronproton pair would look (Figure 8).

We "all have our own time." This means that time is not some fundamental feature, it has to be an effect because we each experience the effect differently. Another way we know that time isn't what we thought it was is that in a quantum state universe time doesn't exist. Time is the comparison of changes between the snapshots. There is no

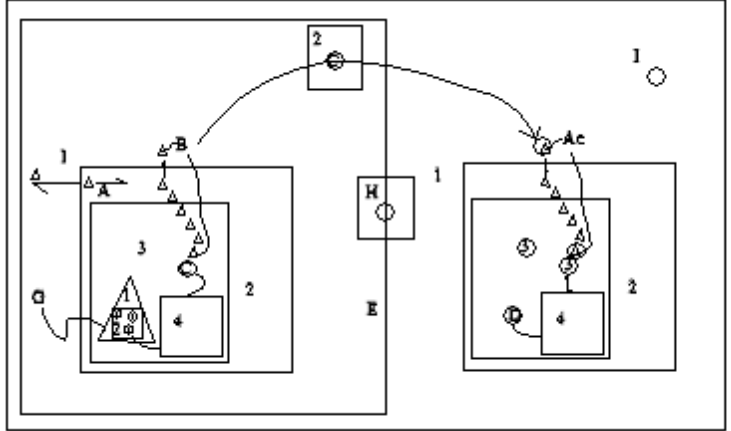

Figure 8: There is 2 neutrons (ct4) surrounded by layers of ct3 (waves), ct2 (photons) and ct1 space. 
time, therefore no thermodynamics, within these snapshots. In each ct state folds at any point a ct 1 charge state can change and if those changes are sufficient for any higher state, the state pulls towards decompression as shown below. Compression is only the opposite effect (Figure 9).

\section{Time 3 Time vs. Dimension and the Time Ratio}

Here is a representative close up of the ct1 movement within a ct3 state. Ct2 states are shown with relative locations. Since all ctl states can rearrange based on the on going solutions two different interactions are shown. Movements are quantum movements in order of ctl rearrangement. While this shows where quantum changes in nondimensional ct 1 end up in a folded matrix of a single ct3, it is important to remember that one dimensional ct2 quantum changes move within a ct3 matrix of ct2 states the same way (Figure 10).

You end up with these streams of different states moving through the matrix of the next higher state and carrying it along as it unfolds. For each neutron you have $1 \times 10^{16}$ quantum wave states, but this translates into $4.3 \times 10^{24} \mathrm{ct} 1$ states which make up that single neutron not including the trapped states which raises this number substantially.

There are massive amounts of ctl in the ct5 matrix, on the scale of $1.5 \times 10^{63}$ paired and perhaps as many again free (quantum points of space).

Transitional states are more dispersed and there are suggestions

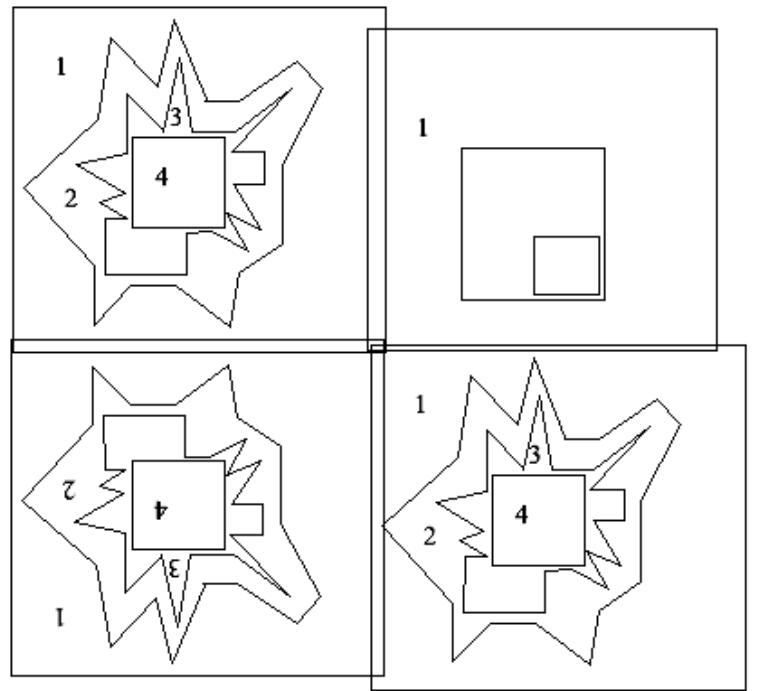

Figure 9: The electron proton pair of helium.
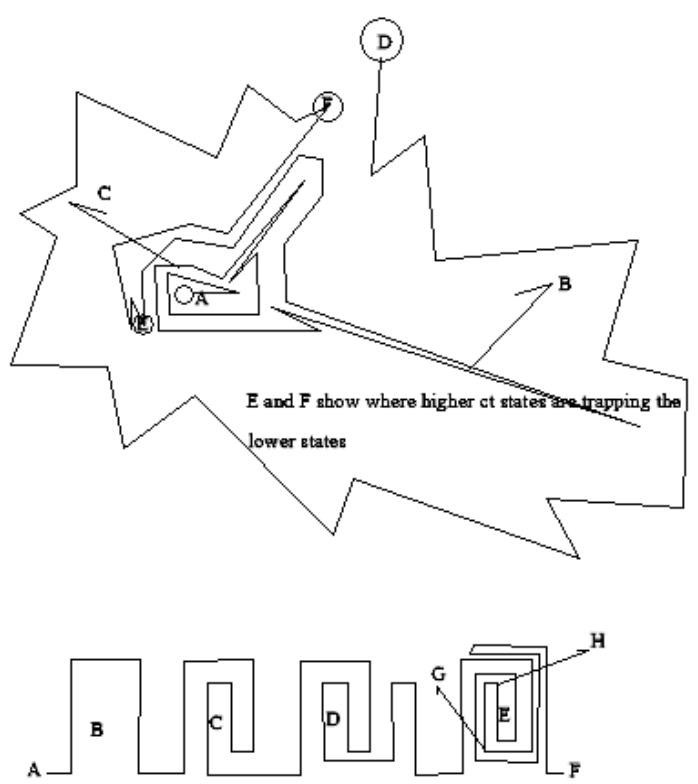

Figure 10: In each ct state folds at any point a ct1 charge state can change and if those changes are sufficient for any higher state, the state pulls towards decompression. 
that the proton has a scale of ct3 states around $5.86 \times 10^{15}$ and the electron $4.4 \times 10^{15}$ states with the free ct 3 states just within the matrix of this pair being perhaps 150 times the number in the electron. As the wavy line of ctl straightens less time occurs because the moving ct1 and 2 changes affect less of the ct 3 within the ct3-4 matrix. As all of the ct2s straighten the entire matrix begins to flatten out. At an inflection point ct 4 breaks down first from neutrons to partially filled states (e.g. electrons and protons) and then into energy (Figure 11).

Those ct 2 changes are electro magnetic changes from the viewpoint of ct304 matrix. In the standard model these are considered to be immature ct 3 photons, but this is because of the confusion around wave particle duality which is cleared up in this model so that photons are one dimensional features with a lower level of compression and the change in alignment between ct 2 and transitional ct 3 is electromagnetism while ct3 (non-transitional) are waves. Since time is a function of ct3 changes within a ct3-4-5 matrix; ct2 EM changes are time independent.

Since ct 1, 2, and 3 exchanges are time independent, gravity can be averaged out (force as ct1 loads onto ct2) over the universe subject to variations of the more compressed pockets. As lines of ct1 pulling on ct2, pulling on ct 3 eventually pull quantum ct 4,3 -dimensional features are pulled towards two dimensional features before they break down.

\section{Range and Strength of Forces Reflecting AuT}

Gravity involves the transition between two different underlying mathematical algorithms, fpix and $2 \mathrm{f}(\mathrm{x})^{2 \mathrm{x}}$ where the fpix solutions are either loaded onto the $2^{2}$ information arms or trapped by the folding resulting from the alternating solutions. All the remaining forces arise from alignment on the information arms of higher states of information arms of a lower state (Figure 12).

1) Strong force: strength 1 range $10^{-15}$. According to AuT this results from compressing or decompressing what we'll call ct4 (neutrons) into what we can call transitional ct5 states which bind the nucleus together.
I use ct 4 instead of mass and ct5 instead of black holes because ct 4 includes some transitional states, electrons and protons, as well as non-transitional neutrons. Ct5 include transitional black hole states which we call molecular states up to the neutron star. The strong force is compression (or decompression) at the ct4-ct5 transitional state we identify as a nucleus (protons and neutrons joined) and hence is limited to the range where protons and neutrons are joined.

2) Weak: $10^{-6}$ Range $10^{-18}$. According to AuT this results from compressing or decompressing wave energy into mass. The Weak force is the compression or decompression at the ct3-ct4 stage and extends to the edge of a proton-electron pair down to neutrons.

3) Electro-magnetic force $1 / 137$ range infinite (AuT-compressed ct2 to a transitional photon or photon to wave) Magnetism, occurs at the ct2-ct3 information arm filling and the wave forces at the ct2-ct3 information arms indicate that stable states are predated by what we see as force and involve $\mathrm{F}(\mathrm{n})^{(2 \mathrm{n})}$ compression or decompression. They result from net compressive math results (CMR) of higher ct states relative lower ct states which are durable from our perspective at this point in the universe. Since these changes are time independent we don not observe them directly and their range which is limited to one dimensional ct 2 to two dimensional ct 3 lengths is not observed.

4) Gravity $6 \times 10^{-39}$ th range infinite (This result comes from space transitioning to photons or ct1 to ct2). Since this transition is both time and dimensionally independent no range is seen or even appropriate.

Folding Gravity, the ct1-ct2 connection creating dimensional linearity from solution order

Gravity results from the interaction of a non-dimensional element (fpix solution space) with (fseries solution) dimensional ct2 (photon) carrier arms.

The range of both gravity and waves is tied to their properties arising from non-dimensional, time independent features of change.

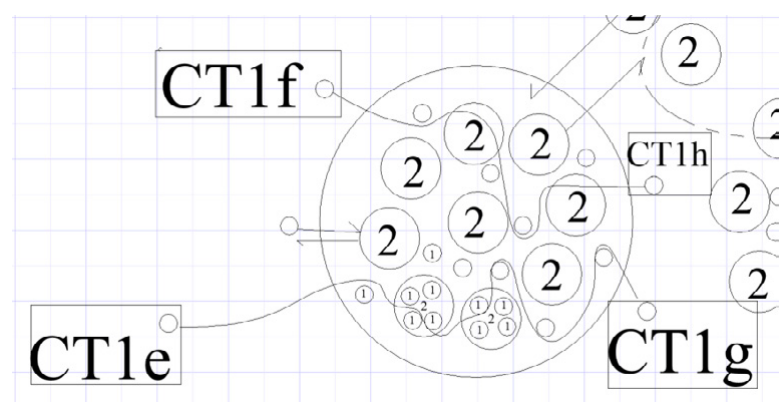

Figure 11: Two different interactions of ct1 states when rearrange based on the ongoing solutions
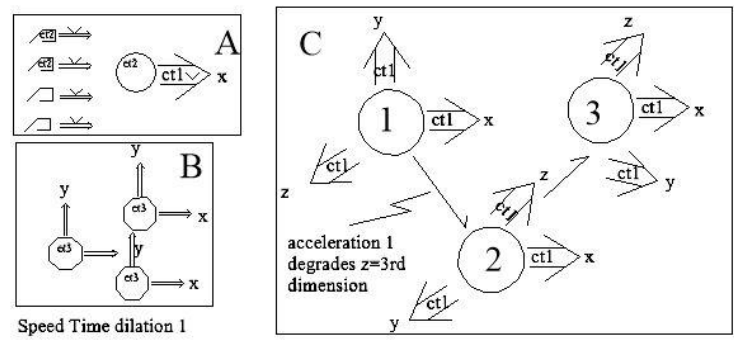

Figure 12: Dimension appears to arise conceptually from increased compression and how it gradually bleeds out compression through velocity. 
While Electromagnetism has dimension, it is unfolded, being one dimensional.

When you get ct1 linearity in the presence of overlaps of ct1 state you get comparative states between ct 1 and ct 2 before time is applied, the result of these folds comparative states between ct 1 and ct 2 before time is applied, the result of these folds is that you experience gravity. Unfolding ct1 from ct2 is dark energy. The idea of folding and unfolding is that as long as the ct 2 states are folding over trapping ct 1 you have gravity. When the ct2 states unfold to release ct1 you have anti-gravity. Both exist together. We experience net gravity, in dark matter the net would be dark energy (Table 6).

The Table above shows the relationship between distance and information exchange.One confusing aspect of this is the implementation of time which is a ct4-ct5 state phenomena looking back at lower ct state exchanges, particularly ct1-ct2 exchange.

The reason this is difficult for us to observe is because we assume that space and photonic energy are different when one is merely the compressed state of the other. In addition, length only occurs relative to this same exchange (and in one dimension). This means the minimum length is an exchange rate 1:256. Time as we experience it, doesn't even exist at this transition because there is no historical point of reference.

\section{Correlations with measured time}

The substitution rate change and seconds can be directly correlated and the other substitution rates can then be worked into the equation (ct2 for ct3, ct3 for ct4, etc to give a precise measurement and reconciling these.

Previously, and somewhat questionably, using $\mathrm{e}=\mathrm{mc}^{2}$ and plank length a minimum quantum time length was calculated as $1.07 \times 10^{-39}$ th of a second.

A more appropriate ratio that allows you to get to the exact number and is it the same as $\mathrm{fn}^{2 \mathrm{n}}$ for $3^{\star} 4$ : $1.6796 \mathrm{E}+22$ ) allow for a solution.

These transitions would occur where the same a single ct1 exchanges with two different groups of ct 4 transitions per 256. Again, this is a discrete change. It is hard to observe only because of the scale at which it happens. A single quantum slow down would be $256 / 46565$ for photon to wave and we have these interchanging which further complicates the analysis.

At the level where we "see" things, ct4, the smallest possible transitions are 256/4,665,600,000,000 ct1 transitions just at the ct4-ct3 interchange. If you accelerate matter to the speed of light then every 256 changes in $\mathrm{x}$ you $1 \mathrm{ct} 1$ interchange or, for a quantum instant in ct 4 you have $18,225,000,000 \mathrm{ct} 1$ exchanges. Theoretically you can change from this to $18,224,999,999 \mathrm{ctl}$ exchanges but I believe the better number is $18,225,000,000-256$ or $18,224,999,744$ changes per quantum instant.

To understand this better it works like this:

1:256 is photon at the speed of light.

Then you have transitions between waves which are $1 \mathrm{ct} 3$ per 46,656 per photon.

Finally, you have the 1:100,000,000 ct4 per wave.

If you use the previously determined and questionable value of a second you get the discreet change between speeds is $256 / 6.6656 \times 10^{11}$ per $1.07 \times 10^{-39}$ th of a second. While discrete, this change in speed is not easily observable. Further, the theorized breakdowns between other states due to fractional transitions would further cloud the analysis. The length of a second is largely a function of ctl exchange (velocity) vs. higher state exchange as discussed above which gives it less relevance.

\section{Conclusion}

\section{Model vs. theory}

AuT is a model, models are different. They take time to build. If you put them together correctly, they are elegant and beautiful, or sometimes scary, but usually reflect the intention of design.

Unlike pre AuT physics, AuT supersymmetry means you cannot have even microscopic symmetry on a molecular scale. Each quantum state may be information, but its length of survival varies preventing a symmetric result. The relationship of one compression state to another

\begin{tabular}{|c|c|c|c|c|c|c|c|}
\hline Information & $2^{*} f(n)$ & $2^{n}$ & $2 f(n)^{2 n}$ & Dim State & & & $f(n)$ \\
\hline \multicolumn{3}{|c|}{ N-CT State } & \multicolumn{5}{|c|}{ Next lower states } \\
\hline 1 & 2 & 2 & 4 & Space-0 & & $2 * 1$ & $0+1$ \\
\hline 2 & 4 & 4 & 256 & Photon-1 & & $2 * 2$ & $1+1$ \\
\hline 3 & 6 & 8 & 1679616 & Wave-2 & $4.30 \mathrm{E}+08$ & $2 * 3$ & $1+2$ \\
\hline 4 & 10 & 16 & $1.00 \mathrm{E}+16$ & Matter-3 & & $2 * 5$ & $2+3$ \\
\hline \multirow[t]{2}{*}{5} & 16 & 32 & $3.40 E+38$ & Black hole-4 & & $2 * 8$ & $3+5$ \\
\hline & & & $3.40 E+38$ & \multicolumn{4}{|c|}{ Ratio of prior state to current state=maximum time } \\
\hline changes/quantum & inst ct4 & & $1.68 \mathrm{E}+22$ & at ct4 & $3 * 4$ & $4.30 \mathrm{E}+24$ & $3 * 4 * 2$ \\
\hline changes/quantum & inst ct5 & & $5.72 \mathrm{E}+60$ & at ct5 & $3 * 4 * 5$ & $1.46 \mathrm{E}+63$ & $3^{*} 4^{*} 5^{\star} 2$ \\
\hline plank length & \multicolumn{2}{|c|}{$1.62 \times 10^{-35}$} & & & 16 & 32 & \\
\hline \multirow[t]{3}{*}{ Light speed } & & $\mathrm{c}=\mathrm{k} / \mathrm{s}$ & $2.99792458 \times 10^{11}$ & $\mathrm{e}=\mathrm{mc}^{2}$ & & & \\
\hline & $3.00 \mathrm{E}+08$ & $\mathrm{~m} / \mathrm{s}$ & & $(\mathrm{e} / \mathrm{m})=\mathrm{c}^{2}$ & & & \\
\hline & $1.60 \mathrm{E}-35$ & Planck & length in meters & 1.62 & $1.00 \mathrm{E}-35$ & & \\
\hline \multicolumn{4}{|c|}{$1.68 \mathrm{E}+22$} & \multicolumn{4}{|c|}{ changes per quantum moment (light speed) } \\
\hline & For a distance & $1.60 \mathrm{E}-35$ & there are & $1.68 \mathrm{E}+22$ & \multicolumn{3}{|c|}{ changes at light speed/quantum length } \\
\hline & & $3.00 \mathrm{E}+08$ & $\mathrm{~m} / \mathrm{s}$ there are & $1.04 \mathrm{E}+57$ & \multicolumn{3}{|c|}{ changes per meter(light speed) } \\
\hline \multicolumn{4}{|c|}{ A quantum instant is $3.46 \mathrm{E}+48$} & \multicolumn{4}{|c|}{ changes per second at ct4 at light speed } \\
\hline \multicolumn{8}{|c|}{ If a quantum moment is $5.40374 \times 10^{-44}$ th of a second (between ct2 and ct4) } \\
\hline \multicolumn{8}{|c|}{ Then the maximum ct4 changes are: } \\
\hline \multicolumn{4}{|c|}{$1.70 \mathrm{E}+22 \mathrm{per}$} & \multicolumn{4}{|c|}{$5.40 \mathrm{E}-44$ of a second at lightspeed } \\
\hline \multicolumn{8}{|c|}{ 5.40E-44 in A Planck length, the portion of a second } \\
\hline
\end{tabular}

Table 6: The relationship between distance and information exchange. 
does not prevent similar states from identical operation, but the length of stability in a given state varies from all others and transitions states along information arms causes even very similar ct states to vary relative to one another.

Supersymmetry in AuT even has a different meaning. It has nothing to do with position and function changing relative to any other point. It means, instead, that you can predict in theory where things were, are or will be with any value of $\mathrm{x}$. An exact proof at later stages of the universe would, of course, be impossible because of the amount of data involved, but at early stages of the universe, these calculations can be made.

\section{References}

1. Friedlander G (2018) Algorithm Universe Theory Book 1-8. CreateSpace Independent Publishing Platform, US, pp: 304.

2. Friedlander (2018) AuM Summary Review of AuT Compendium.

3. Friedlander (2013-2018) Algorithm Universe Theory. (2ndedn), Createspace Independent Publishing Platform, US.

4. Friedlander $G$ (2017) An Overview of the origin of space time and quantum phenomena. Createspace Independent Publishing Platform, US, pp: 132.
5. Friedlander (2018) Mathematical Proofs of Supersymmetry. (5thedn), Createspace Independent Publishing Platform, US.

6. Friedlander (2018) Transition from non-dimensional phenomena to space time. (4thedn), Createspace Independent Publishing Platform, US.

7. Friedlander (2018) State Dimension and Time. (2ndedn), Createspace Independent Publishing Platform, US.

8. Friedlander (2018) Dimension, Time and logic.

9. Friedlander (2018) AuT and the Standard Model.

10. Friedlander (2018) AuT The nature of time as electron movement. Createspace Independent Publishing Platform, US, pp: 162.

11. Friedlander G (2016) Spirals in Amber, The single variable solution to the universe and the origin of quantum mechanics. (2nd edn), Createspace Independent Publishing Platform, US, pp: 284.

12. Friedlander (2016) A Spiral in Amber-The origin of Quantum Mechanics.

13. Friedlander G (2014) Non linear time theory, successor to the Einstein Hologram Universe. Createspace Independent Publishing Platform, US, pp: 152.

14. Friedlander (2018) Conference on Astrophysics and Particle Physics in Chicago, A model for generating dimension and time.. 